\title{
EXISTE CORRELAÇÃO ENTRE QUALIDADE DE VIDA E NÍVEL DE DEPENDÊNCIA DE NICOTINA EM TABAGISTAS?
}

\begin{abstract}
Emanuel Hitalo de Araújo Novaes*, Gabriela Lago Rosier**, Marina Lemos Carvalho Silva***, Maristela Rodrigues Sestelo***, Luciana Bilitário Macedo*****, Aquiles Assunção Camelier******, Cristiane Maria Carvalho Costa Dias ${ }^{* * * * * * *}$
\end{abstract}

Autor correspondente: Luciana Bilitário Macedo - lucianabilitario@bahiana.edu.br

* Acadêmico do 80 semestre do Curso de Fisioterapia da Escola Bahiana de Medicina e Saúde Pública; Bolsista de Iniciação Científica da FAPESB; Membro do Grupo de Pesquisa em Fisioterapia Cardiovascular e Respiratória da Bahiana (GEPFIR)

** Acadêmica do 90 semestre do Curso de Fisioterapia da Escola Bahiana de Medicina e Saúde Pública; Bolsista de Iniciação Científica do PIBIC; Membro do Grupo de Pesquisa em Fisioterapia Cardiovascular e Respiratória da Bahiana (GEPFIR)

*** Acadêmica do 90 semestre do Curso de Fisioterapia da Escola Bahiana de Medicina e Saúde Pública; Bolsista de Iniciação Científica da FAPESB; Membro do Grupo de Pesquisa em Fisioterapia Cardiovascular e Respiratória da Bahiana (GEPFIR)

**** Professora Assistente da Escola Bahiana de Medicina e Saúde Pública

***** Professora Assistente da Escola Bahiana de Medicina e Saúde Pública; Docente da Universidade do Estado da Bahia; Membro do Grupo de Pesquisa em Fisioterapia Cardiovascular e Respiratória da Bahiana (GEPFIR).

****** Professor Adjunto da Escola Bahiana de Medicina e Saúde Pública; Docente da Universidade do Estado da Bahia

******* Professora Adjunta da Escola Bahiana de Medicina e Saúde Pública; Membro do Grupo de Pesquisa em Fisioterapia Cardiovascular e Respiratória da Bahiana (GEPFIR)

\section{Resumo}

Introdução: O consumo de tabaco predispõe a doenças, incapacidade funcional e alta morbimortalidade, resultando em impacto na qualidade de vida (QV) da população em geral. A QV é um tema importante e a constatação de sua associação com o nível de dependência de nicotina pode ser um caminho para sensibilizar os dependentes dessa substância a interromperem o consumo. Objetivo: Verificar se existe correlação entre a qualidade de vida e o nível de dependência de nicotina em tabagistas. Materiais e Métodos: Estudo transversal, constituído por tabagistas admitidos no programa "Deixando de Fumar Sem Mistérios" da Escola Bahiana de Medicina e Saúde Pública, de ambos os sexos e idade igual ou superior a 18 anos. Excluídos tabagistas com dificuldade de compreensão dos questionários. Aplicados questionários validados:Tolerância de Fagerstrom e Whoqol-Bref. Resultados: As características sociodemográficas e clinicas dos 64 participantes estudados demonstraram

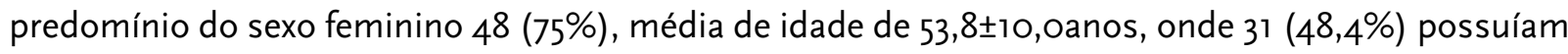
ensino médio e dois $(3,1 \%)$ eram analfabetos. Em relação ao número de maços de cigarros consumidos por ano, a mediana foi de 34,37(21-49) maços/ano. E mediana igual a 2,0(2,0-4,0) na classificação da dependência de nicotina segundo a escala de Fagerstrom, traduzindo em uma baixa dependência. 
A análise inferência do Whoqol-Bref e o nível de dependência de nicotina não revelou correlação com os domínios físico, psicológico, social e ambiental, nem com a pontuação total de QV. Conclusão:O presente estudo não evidenciou uma correlação entre a QV e o nível de dependência de nicotina, bem como da carga tabágica.

Palavras-chave: Tabaco; Qualidade de vida; Dependência a nicotina.

\title{
IS THERE CORRELATION BETWEEN QUALITY OF LIFE AND NICOTINE DEPENDENCE LEVEL IN SMOKERS?
}

\begin{abstract}
Introduction: The consumption of tobacco predisposes to disease, functional disability and high morbidity and mortality rates, resulting in an impact on quality of life (QOL) of the population in general. The QOL is an important topic and the fact its association with the level of nicotine dependence can be a way to sensitize the dependent on that substance to discontinue the consumption. Objective: To correlate the quality of life and the level of nicotine dependence in smokers. Materials and Methods: Cross-sectional study, consisting of smokers admitted to the program "Quitting Smoking Without Misteries" EBMSP / Brotas Unit, both sexes and age $\geq 18$ years. Smokers with limited understanding of the questionnaires were excluded from the sample. The following validated questionnaires were applied: Fagerstrom Tolerance and Whoqol-Brief. Results: The sociodemographic characteristics and clinical of 64 participants studied showed a predominance of females $48(75 \%)$, average age of $53.8 \pm 10.0$ years, where 31 (48.4\%) had a high-school and two (3.1\%) were illiterate. In relation to the number of packs of cigarettes smoked per year, the median was 34.37(21-49) sets/year. And a median of $2.0(2,0-4,0)$ in the classification of nicotine dependence according to the scale of Fagerstom, translating into a lower dependence. The analysis of inference Whoqol-Bref and the level of nicotine dependence has not revealed a correlation with the domains physical, psychological, social and environmental development, nor with the total score of QOL. Conclusion: This study did not show a significant correlation between QOL and the level of nicotine dependence, as well as smoking history.
\end{abstract}

Kepwords: Tobacco; Quality of life; Addiction to nicotine.

\section{INTRODUÇÃO}

O consumo do tabaco está relacionado a maior predisposição para doenças, incapacidades e alta morbimortalidade, resultando em impacto no estilo e qualidade de vida $(\mathrm{QV})$ da população em geral.(1-4) Além das consequências negativas relacionadas à saúde, o tabagismo provoca enormes custos sociais, econômicos e ambientais. Em países desenvolvidos os gastos totais com a saúde consomem de $6 \%$ a 15\% da renda relacionados aos cuidados com as doenças associadas ao tabagismo, chegando a ser o dobro 
nos países em desenvolvimento. ${ }^{(3-6,8)}$ É reconhecido como uma dependência química que expõe os indivíduos à inúmeras substâncias tóxicas. ${ }^{(5)}$ Sendo classificado internacionalmente pela Organização Mundial de Saúde (OMS) no grupo dos transtornos mentais e de comportamento (CID 10) decorrentes do uso de substâncias psicoativas. . $^{(6,7)}$

Devido ao elevado índice de consumo de cigarro, seus usuários se tornam mais susceptíveis às doenças relacionadas ao tabaco, tais como o câncer, doenças coronárias, pulmonares, bem como a impacto na QV. Tem sido observado um aumento no número de estudos mensurando à $\mathrm{QV}$ dos tabagistas. Um ponto em comum encontrado nessas pesquisas é o impacto negativo na $\mathrm{QV}$ dos tabagistas, quando comparados aos não tabagistas. Isto significa que a dependência da nicotina interfere na QV dos fumantes diretamente ou indiretamente, mesmo considerando um constructo subjetivo e multidimensional. . $^{(-6,8,9)}$

Dados epidemiológicos revelam que em 2005, $35,9 \%$ das internações de homens e $27 \%$ das de mulheres foram atribuídas ao tabagismo pelo Sistema Único de Saúde (SUS), sendo necessário apoio aos fumantes. Segundo a OMS, abandonar do hábito de fumar é cada vez mais evidente, chegando a $68 \%$ o número de fumantes que procuram apoio, estimulados por um ambiente social cada vez mais desfavorável ao consumo do tabaco e diante das doenças relacionadas ao mesmo. ${ }^{(8,9-12)}$

O programa Deixando de fumar sem mistérios, de iniciativa do Ministério da Saúde, tem como objetivo oferecer tratamento para cessação tabágica baseada no apoio e terapia a grupos e uso de farmacológicos, bem como na sua reeducação, enfocando os benefícios de não fumar para a manutenção da saúde, melhora do estilo e QV, redução da morbidade e das incapacidades decorrentes do consumo do tabaco, e ainda na diminuição dos gastos com a saúde pública.

O uso do tabaco é considerado uma ameaça ao desenvolvimento econômico e social, tendo em vista a diminuição da produtividade, especialmente da população com idade economicamente ati- va. ${ }^{(1-6)}$ Há de se observar o impacto direto na vida econômica do fumante, ou seja, no quanto a sua dependência à substância "nicotina", desencadeia um baixo rendimento, seja a nível laboral, econômico/familiar, e de expectativa de vida. Sendo assim, o presente estudo tem como o presente estudo tem como objetivo verificar se existe correlação entre a qualidade de vida e o nível de dependência de nicotina em tabagistas.

\section{MATERIAIS E MÉTODOS}

Trata-se de um estudo observacional de caráter transversal, CAAE: 37684014.2.0000.5544. Todos os participantes do estudo assinaram o Termo de Consentimento Livre e Esclarecido (TCLE). A amostra foi composta por 64 participantes, na qual a população acessível consistiu em tabagistas admitidos no programa Deixando de fumar sem Mistérios na Escola Bahiana de Medicina e Saúde Pública (EBMSP) - Unidade Brotas, ambos os sexos e idade $\geq 18$ anos. Excluídos os tabagistas com déficit de compreensão dos questionários.

O cálculo amostral foi realizado através da calculadora WinPepi (publichealth.jbpub.com/book/ gerstman/winpepi.cfm). O comando selecionado foi de Etcetera, coeficiente de correlação adotado foi de 0,4 , com poder de teste de $90 \%$, com nível de significância de $5 \%$, necessitando de 62 indivíduos.

A coleta de dados foi realizada no período de juIho de 2014 a agosto de 2015 , por pesquisadores treinados; aplicado o questionário sócio demográfico contendo o perfil de consumo de tabaco e outras substâncias psicoativas, dados antropométricos e comorbidades autorreferidas. Em seguida aplicou-se o questionário de Tolerância de Fagerstrom, para avaliar o nível de dependência de nicotina, que é um instrumento amplamente utilizado nesta população e validado no Brasil. Consiste em seis questões, duas pontuadas de zero a três e as demais de zero a um, sendo o indivíduo classificado como: muito baixa dependência: o a 2 pontos; baixa dependência: 3 e 4 pontos; média dependência 5 pon- 
tos; elevada dependência: 6 e 7 pontos, ou muito elevada dependência 8 a 10 pontos. ${ }^{(14)}$

O questionário Whoqol-BREF foi elaborado pela OMS e validado no Brasil, com objetivo de avaliar a qualidade de vida. Consiste em 26 itens abrangendo quatro domínios: físico, psicológico, social e ambiental, uma questão relativa à qualidade de vida geral e, outra à satisfação com a saúde, onde as respostas são baseadas na escala de Likert. ${ }^{(1,15)}$

Para análise estatística foi utilizado o Statistical Package for Social Sciences (SPSS) versão 14.0 para Windows. As variáveis categóricas foram expressas em valores absolutos e relativos. $\mathrm{E}$ as variáveis numéricas com distribuição simétrica foram expressas em média desvio padrão e distribuição assimétrica expressas em mediana e intervalo interquartil. A normalidade das variáveis foi verificada através do teste de normalidade Kolmogorov - Smirnov e análise descritiva, considerando a descritiva soberana em caso de discordância Os resultados apresentados por meio de tabelas e figuras. Para análise inferencial foi utilizado o teste coeficiente correlação de Pearson aplicada na mensuração dos domínios do questionário qualidade de vida e nível de dependência de nicotina, representada através dos resultados do questionário tolerância de Fagerstrom e carga tabágica (marcos/ ano). O nível de significância adotado foi de $5 \%$.

\section{RESULTADOS}

As características sociodemográficas e clinicas dos 64 participantes demonstraram uma predominância do sexo feminino com 48 (75\%), média

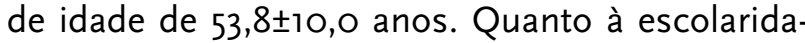
de, $31(48,4 \%)$ possuíam ensino médio completo e dois $(3,1 \%)$ eram analfabetos. O tempo de uso de tabaco nesta população teve uma média de $36,63 \pm 11,8$ anos. O questionário de questionário de Tolerância de Fagerstrom apresentou uma mediana igual a 2,0 (2,0-4,0), traduzindo uma baixa dependência à nicotina. Em relação ao número de maços de cigarros consumidos por ano, a mediana foi de 34,37 (21-49) maços/ano, tabela 1.

Tabela 1 - Características sociodemográficas e antropométricas dos participantes do programa Deixando de fumar sem Mistérios. $\mathrm{N}=64$. Salvador/BA, 2016

\begin{tabular}{|c|c|}
\hline VARIÁVEIS & MÉDIA \pm DP \\
\hline Idade & $53,84 \pm 10,0$ \\
\hline Maço/Ano & $* 34,37$ (21-49) \\
\hline Dependência & $* 2, \mathrm{O}(2, \mathrm{O}-4, \mathrm{O})$ \\
\hline VARIÁVEIS & N (\%) \\
\hline \multicolumn{2}{|l|}{ Sexo } \\
\hline Feminino & $48(75,0)$ \\
\hline \multicolumn{2}{|l|}{ Escolaridade } \\
\hline Analfabetos & $2(3,1)$ \\
\hline Ensino fundamental & $16(25,0)$ \\
\hline Ensino médio & $31(48,4)$ \\
\hline Ensino superior & $15(23,4)$ \\
\hline \multicolumn{2}{|l|}{ IMC } \\
\hline Eutrófico & $29(45,3)$ \\
\hline Sobrepeso & $28(43,8)$ \\
\hline Obesidade & $7(10,9)$ \\
\hline
\end{tabular}

IMC - Índice de Massa Corporal. DP= desvio padrão. * Resultado expresso em mediana e Intervalo Interquartil(IQ). 
Dentre as doenças autorreferidas, a Hipertensão Arterial Sistêmica (HAS) foi evidenciada em $23(35,4 \%)$ dos participantes, e as demais doenças, obteve-se uma baixa frequência nesta população, como: dislipidemia $5(7,8 \%)$, diabetes mielites
$11(17,2 \%)$, doenças pulmonares $8(12,5 \%)$ e doenças cardíacas $4(6,3 \%)$.

A tabela 2 demonstra ausência de correlação dos domínios do Whoqol-Bref com a dependência de nicotina e a carga tabágica dos indivíduos através do teste de correlação de Pearson.

Tabela 2 - Correlação dos domínios do Whoqol-BREF com a dependência de nicotina e a carga tabágica. N=64. Salvador/BA, 2016

\begin{tabular}{lcccc}
\hline \multicolumn{1}{c}{ Domínios } & \multicolumn{2}{c}{ DePENDÊNCIA DE Nicotina** } & \multicolumn{2}{c}{ MARÇOS/ANO } \\
\hline Físico & $\mathbf{R}$ & $\mathbf{P}$ & $\mathbf{R}$ & $\mathbf{p}$ \\
Psicológico & $-0,20$ & 0,10 & $-0,02$ & 0,87 \\
Social & $-0,03$ & 0,79 & 0,02 & 0,84 \\
Ambiental & $-0,17$ & 0,16 & $-0,04$ & 0,72 \\
Total & $-0,09$ & 0,43 & 0,00 & 0,97 \\
\hline
\end{tabular}

* Teste de correlação de Pearson ** Avaliado pelo questionárioWhoqol-Bref

Segundo o questionário de tolerância Fagerstrom, o nível de dependência à nicotina nesta população foi predominantemente baixo, com uma frequência de 24 (37,5\%), sendo sete $(10,9 \%)$ classificados com dependência muita elevada, figura 1.

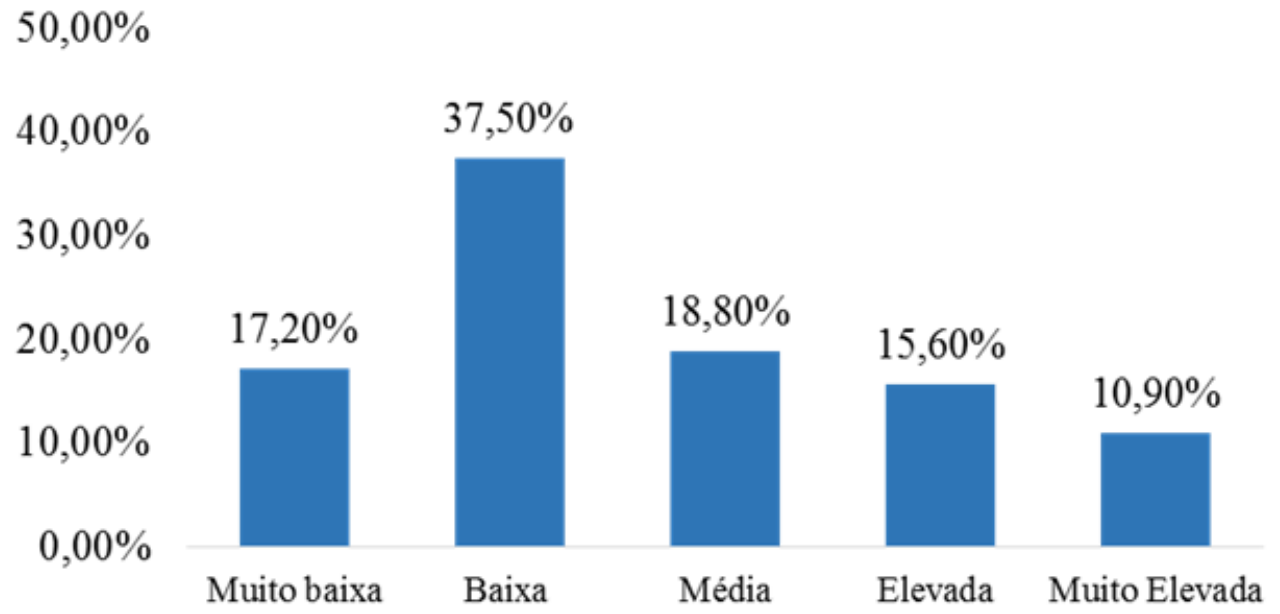

Figura 1 - Frequência das categorias do questionário tolerância de Fagerstrom dos participantes do programa "Deixando de fumar sem Mistérios". N=64. Salvador/BA, 2016 


\section{DISCUSSÃO}

O presente estudo não revelou correlação entre a QV e o nível de dependência de nicotina, nem com a carga tabágica, representada pelos marços de cigarros por ano.

Não houve impacto nos domínios físico, psicológico, social, ambiental do questionário Whoqol-Bref, o qual avalia a percepção do indivíduo acerca da QV, apresentou uma média de $56,39 \%$ no escore qualidade de vida geral, refletindo uma razoável percepção. Esses resultados podem estar relacionados ao perfil sociodemográfico e clinico dessa população, ressaltando a baixa incidência de doenças associadas, o que corrobora com outros autores que mostraram uma relação da piora da $\mathrm{QV}$ com a presença de comorbidades associadas, tais como uma maior faixa etária, baixa escolaridade e nível socioeconômico e o sexo. ${ }^{(1-5,7,7,9)}$

Observou-se um maior predomínio do sexo feminino, constituída por uma população considerada adulta jovem e com grau de escolaridade maior, que buscaram tratamento para a cessação do tabagismo no programa "Deixando de fumar sem mistérios". O predomínio de mulheres em programas que abordam a cessação do tabagismo tem sido descrito, pois nestes, são prestados aconselhamentos médicos com maior frequência, além do fato de que estas acreditam e se preocupam com o fato de que o tabagismo causa câncer de diversas etiologias e provoca o surgimento de doenças pulmonares e cardiovasculares. . $1-4,6,8,13-20)^{-1}$

Estudos apontam que a dependência de tabaco está associada a vários fatores, tais como escolaridade e nível socioeconômico. No Brasil as pessoas com baixa escolaridade têm uma probabilidade cinco vezes maior de serem fumantes e o consumo de cigarros é maior nas classes de menor renda. ${ }^{(7-9,10,12)}$ Em contrapartida nessa pesquisa, a maioria dos participantes possuía ensino médio e superior, revelando que não somente os de menor escolaridade fumam nos dias atuais. Esse dado pode refletir também uma menor adesão das pessoas com baixa renda aos programas devido ao não acesso a informação de forma ampla e adequada da existência dos mesmos.

De acordo com a média de idade e o tempo médio de uso do tabaco da população, os autores entendem que o hábito de fumar e a dependência à nicotina se estabeleceu na adolescência, corroborando com a literatura. ${ }^{(4,5)}$ Esse hábito tão precoce tem uma implicação importante a longo prazo na saúde individual e pública, principalmente na qualidade de vida. A procura de jovens por um programa de apoio a cessação tabágica, tem como finalidade a sua reinserção na sociedade, uma vez que, tal postura não é vista mais como uma tendência atual, como foi na década de 80 .

Além disso, foi observado que a maioria dos participantes eram eutróficos. Alguns autores afirmam que fumantes têm menor IMC do que não fumantes. ${ }^{(5,6,6)}$ Corroborando com esses achados, outros autores acrescentaram que fumantes pesam em geral menos do que os não fumantes, e ganham peso quando param de fumar, em decorrência da ação da nicotina no indivíduo. ${ }^{(3,8,11,16,18)}$

A baixa frequência das doenças associadas nesta população pode ser justificada por se tratar de uma população adulta jovem, predominantemente do sexo feminino o que pode traduzir uma maior frequência no cuidado com a saúde. ${ }^{(8-13,20)}$ Outro aspecto a ser considerado foi a auto referência das comorbidades, que pode refletir sub diagnóstico ou ausência de sintomatologias associadas às comorbidades crônicas.

Esse estudo apresenta como limitação o seu caráter transversal, sem a comparação da QV no início e no final do programa. Além disso, há carência de estudos nacionais e internacionais sobre o instrumento utilizado (Whoqol-Bref) que apontem o ponto de corte que determine escore indicando as categorias para classificar a QV. Novas pesquisas de intervenção objetivando preencher as lacunas existentes devem ser estimuladas. 


\section{CONCLUSÃO}

O presente estudo não evidenciou uma correlação entre QV e o nível de dependência de nicotina e carga tabágica.

\section{AGRADECIMENTOS}

Em memória de Emanuel Hitalo de Araújo Novaes que mostrou que com persistência e coragem podemos alcançar todos nossos objetivos.

Este artigo é parte da tese do doutorado de Luciana Bilitário Macedo no programa de Pós Graduação em Medicina e Saúde Humana da Escola Bahiana de Medicina e Saúde Pública.

\section{REFERÊNCIAS}

1. Castro MDG, Oliveira MDS, Moraes JFD, Miguel AC, Araujo RB. Qualidade de vida e gravidade da dependência de tabaco. Revista de Psiquiatria Clinica. 2007;34:61-67. DOI: 10.1590/SO101-60832007000200001

2. Castro MRP, Matsuo T, Nunes SOV.

Características clínicas e qualidade de vida de fumantes em um centro de referência de abordagem e tratamento do tabagismo. J Bras Pneumol. 2009;36(1):67-74.

3. Dantas RAS, Sawada NO, Malerbo MB. Pesquisas sobre qualidade de vida:revisão da produção científica das universidades públicas do Estado de São Paulo. Rev Latinoam Enferm. 2003;11(4):532-538. DOI: 10.159O/SO1O411692003000400017

4. Marques ACPR, Campana A, Gigliotti AP, Lourenço MTC, Ferreira MP, Laranjeira R. Consenso sobre o tratamento da dependência de nicotina. Rev Bras Psiquiatr. 2001;23(4):200-14.

5. Araújo AJ, Menezes AMB, Dórea AJPS, Torres BS, Viegas CAA, Silva CAR, et al. Diretrizes para Cessação do Tabagismo. J. bras. pneumol. [Internet]. 2004;30( Suppl 2 ):S1-S76. Available from: http://www.scielo. $\mathrm{br} / \mathrm{scielo}$.php?script=sci_arttext\&pid=S180637132004000800002\&lng=en. http://dx.doi. org/10.1590/S1806-37132004000800002

6. Jenner T, Falc O, Costa C. O tabagismo em um municipio de pequeno porte: um estudo etnográfico como base para geração de um programa de saúde pública. J Bras Pneumol. 2008;34(2):91-7.

7. Tomioka H, Sekiya R, Nishio C, Ishimoto G. Impact of smoking cessation therapy on health-related quality of life. BMJ Open Respir Res. [Internet]. 2014;1(1):1-11. DOI: 10.1136/ bmjresp-2014-000047

8. Macedo LB, Araújo CBS, Dias CMCC. Efeitos dos programas educacionais em pacientes com asma: revisão sistemática. ASSOBRAFIR Ciência. 2012;3(2):43-52.

9. Organização Mundial de Saúde. Tabagismo \& Saúde nos Países em Desenvolvimento. 2003. p. 1-15.

10. Sales MPU, Oliveira MI, Mattos IM, Viana CMS, Pereira EDB. Impacto da cessação tabágica na qualidade de vida dos pacientes. J Bras Pneumol. 2009;35(5):436-41.

11. Santana VTS, Squassoni SD, Neder JA, Fiss E. Influência do tabagismo atual na aderência e nas respostas à reabilitação pulmonar em pacientes com DPOC. Rev Bras Fisioter. 2O10;14(1):16-23. DOI: 10.1590/S141335552010000100004

12. Santos LLAG, Ormond LDS, Macedo MC, Dias CMCC, Macedo LB. Sinais e sintomas respiratórios, grau de dependência ao fumo e nível de atividade física em tabagistas. ASSOBRAFIR Ciência. 2013;4(2):7-37.

13. Tillmann M, Silcock J. A comparison of smokers' and ex-smokers' health-related quality of life. J Public Health Med. 1997;19(3):268-273.

14. Barbisan JN, Manfroi WC. Utilização do teste de dependência à nicotina de Fagerstrom como um instrumento de medida do grau de dependência. Rev HCPA. 2007;3(27):31-6.

15. Fleck MPA, Louzada S, Xavier M, Chachamovich E, Santos L, Fleck MPA et al. Aplicação da versão em português do instrumento abreviado de avaliação da quali- dade de 
vida "WHOQOL-bref." Rev Saúde Pública. 2000;34(2):178-83.

16. Arriaga EEB, Oliver PV, Alejandra R, Sansores $\mathrm{RH}$. Efecto del daño de vías dopaminérgicas mesencefálicas en la conducta adictiva al tabaco. Revista Del Instituto Nacional de Enfermedades Respiratorias Ismael Cosio Villegas. 2007;2O(1):56-63.

17. Furtado RD. Implicações Anestésicas do Tabagismo. Rev Bras Anestesiol. 2002; 354-67. DOI: 10.1590/SO034-70942002000300012
18. Pereira A, Balbani S, Montovani JC. Métodos para abandono do tabagismo e tratamento da dependência da nicotina. Rev Bras Otorrinolaringol. 2005;71(6):820-7. DOI: 10.1590/SO034-72992005000600021

19. Planeta CS, Cruz FC. Bases neurofisiológicas da dependência do tabaco. Rev. Psiq. Clín. 2005;32(12):251-258.

2O. Rodrigues C, Santos SRA, Hetem LA, Dias JC, Naka MU, Quintino MP, et al. Diretrizes em foco - Tabagismo. Rev Assoc Med Bras. 2010;2(2):134-7. 